\title{
Influence of sumatriptan on gastric fundus tone and on the perception of gastric distension in man
}

\author{
J Tack, B Coulie, A Wilmer, A Andrioli, J Janssens
}

\begin{abstract}
Background-In animals, activation of 5-HT 1 like receptors causes a relaxation of the gastric fundus through the activation of intrinsic inhibitory neurones.

Aims-To investigate the effect of sumatriptan, an agonist at enteric neuronal 5-HT 1 receptors, on fasting fundus tone and sensitivity to gastric distension in man.

Methods-A gastric barostat was used to study the effect of placebo and sumatriptan, $6 \mathrm{mg}$ subcutaneously, on basal fundic tone in healthy subjects. In addition, stepwise isobaric and isovolumetric gastric distensions were performed and perception was measured before and after the administration of placebo and sumatriptan.
\end{abstract}

Results-Placebo had no significant effects on gastric tone and on perception. Sumatriptan induced an immediate relaxation of the gastric fundus, reflected by an intragastric volume increase of 209 (39) $\mathrm{ml}(\mathrm{p}<0.0005)$. After sumatriptan, intragastric pressures at the thresholds for perception or discomfort were not significantly altered. However, the intragastric volumes and the corresponding calculated wall tensions at perception and discomfort thresholds were significantly increased. Conclusions-Administration of the 5-HT receptor agonist sumatriptan induces a relaxation of the gastric fundus in man, allowing larger intragastric volumes before thresholds for perception or discomfort are reached. The effects of sumatriptan on the gastric fundus may have therapeutic potential in the treatment of patients with functional dyspepsia.

(Gut 2000;46:468-473)

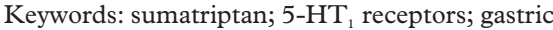
barostat; visceral sensitivity; enteric nervous system.

During fasting, gastric fundus tone in the dog is maintained by a vagally mediated cholinergic input. ${ }^{1}$ After a meal, a receptive relaxation of the fundus occurs, which is mediated via a vagovagal reflex pathway, and which activates non-adrenergic non-cholinergic neurones in the gastric wall. ${ }^{23}$ In the mouse and in the guinea pig, involvement of 5-hydroxytryptamine (5-HT) receptors on intrinsic neurones in the vagally mediated gastric relaxation has been shown. ${ }^{4}$ More recently, it was shown that 5-HT induced relaxations of the guinea pig stomach are mediated via the release of nitric oxide through activation of a $5-\mathrm{HT}_{1}$ like receptor. ${ }^{5}$

In man, the effect of 5-HT on gastric fundus tone has not been studied directly. Several subtypes of 5-HT receptors are known. ${ }^{6}$ Of these, $5-\mathrm{HT}_{1 \mathrm{~A}}, 5-\mathrm{HT}_{1 \mathrm{P}}$, and $5-\mathrm{HT}_{3}$ receptors have been identified in the myenteric plexus of the guinea pig stomach. ${ }^{7}$ Selective antagonism of $5-\mathrm{HT}_{3}$ receptors by ondansetron or alosetron does not influence interdigestive and postprandial fundus tone in man. ${ }^{8}$ Lack of suitable ligands precluded a similar study for $5-\mathrm{HT}_{1 \mathrm{P}}$ receptors. Recently, however, we showed that sumatriptan, a 5-HT ${ }_{1}$ receptor agonist which is used in the treatment of migraine in man, ${ }^{10}$ is an agonist at $5-\mathrm{HT}_{1 \mathrm{P}}$ receptors on nitrergic myenteric neurones in the stomach. ${ }^{11}$

The present study was undertaken to test the hypothesis that sumatriptan is able to induce a relaxation of the gastric fundus in man. An electronic barostat was used to register variations in gastric fundus tone in healthy volunteers before and after the administration of placebo or sumatriptan. The effects of placebo and sumatriptan on gastric compliance and on the perception of gastric distension were also assessed.

\section{Materials and methods}

STUDY SUBJECTS

Fifty eight healthy volunteers (36 men and 22 women; aged 19-30 years; mean age 23.4 (0.4) years) participated in this study. None of the subjects had symptoms or a history of gastrointestinal disease or drug allergies, nor were they taking any medication. Informed consent was obtained from each participant. The protocol was approved by the Ethics Committee of the University Hospital.

\section{RECORDING TECHNIQUE}

Following an overnight fast of at least 12 hours, a double lumen polyvinyl tube (Salem sump tube $14 \mathrm{Ch}$, Sherwood Medical, Petit Rechain, Belgium) with an adherent plastic bag (1200 $\mathrm{ml}$ capacity; $17 \mathrm{~cm}$ maximal diameter) finely folded, was introduced through the mouth and secured to the subject's chin with adhesive tape. The position of the bag in the gastric fundus was checked fluoroscopically.

The polyvinyl tube was then connected to a computer driven programmable volume displacement barostat device (Synectics Visceral Stimulator, Stockholm, Sweden). The barostat device can deliver volume ramps or pressure

Abbreviations used in this paper: $5-\mathrm{HT}$, 5-hydroxytryptamine; MDP, minimal distending pressure; MI, motility index. 
steps at different rates, while simultaneously monitoring pressure and volume at a sampling rate of eight samples per second. To unfold the intragastric bag, it was inflated with a fixed volume of $500 \mathrm{ml}$ of air for two minutes with the study subject in a recumbent position, and again deflated completely. After a 10 minute equilibration period, the subjects were positioned in a comfortable sitting position with the knees slightly bent $\left(80^{\circ}\right)$ in a bed, specifically designed for that purpose.

\section{STUDY DESIGN}

The study consisted of two separate protocols. In the first protocol, the acute effects of placebo (subcutaneous saline) $(n=10)$ or sumatriptan $(n=11)$ on basal volume were studied in 21 subjects (13 men and eight women, age range 19-30 years). After a 30 minute accommodation period, minimal intragastric distending pressure (MDP) was first determined as the lowest pressure level that provided an intrabag volume of $30 \mathrm{ml}$ or more. ${ }^{12}$ This pressure level equilibrates the intra-abdominal pressure. With the subjects in sitting position, MDP, determined by increasing intrabag pressure by $1 \mathrm{~mm} \mathrm{Hg}$ every three minutes, was $6.7(0.4)$ $\mathrm{mm} \mathrm{Hg}$. During the study, a fixed intrabag pressure of $2 \mathrm{~mm} \mathrm{Hg}$ above MDP was set, and the intrabag volume at this pressure was recorded during 45 minutes after which placebo or sumatriptan (Imitrex, Glaxo Wellcome, Belgium) $6 \mathrm{mg}$ was administered subcutaneously. The recording of the intrabag volume at $\mathrm{MDP}+2 \mathrm{~mm} \mathrm{Hg}$ continued for another 45 minutes.

In the second protocol, after a 30 minute accommodation period, a sequence of stepwise isobaric or isovolumetric distensions were performed. The MDP was again defined as the lowest pressure level that provided an intrabag volume of $30 \mathrm{ml}$ or more. Subsequently, the pressure level was set at MDP $+2 \mathrm{~mm} \mathrm{Hg}$ during 30 minutes, with administration of sumatriptan $6 \mathrm{mg}$ or placebo subcutaneously after 15 minutes. Afterwards, graded distensions were repeated. Subjects were instructed to score their perception of upper abdominal sensations induced by each distending stimulus at the end of every distending step, using a graphic rating scale that combined verbal descriptors on a scale graded from 0 to $6 .{ }^{12}$ The end point of each sequence of distensions was established at an intrabag volume of $1000 \mathrm{ml}$, or when the subjects reported discomfort or pain (score 5 or 6 ).

In 23 subjects ( 15 men and eight women), the effect of placebo $(n=10)$ or sumatriptan $(n=13)$ on the perception of isobaric gastric distensions was evaluated. Sequential distensions were performed in stepwise increments of $2 \mathrm{~mm} \mathrm{Hg}$ starting from MDP, each lasting for two minutes, while the corresponding intragastric volume was recorded. In 14 subjects (eight men and six women), the effect of placebo $(n=7)$ or sumatriptan $(n=7)$ on the perception of isovolumetric gastric distensions was evaluated. Sequential distensions were performed in stepwise increments of $100 \mathrm{ml}$ starting from $100 \mathrm{ml}$, each lasting for two minutes, while the corresponding intragastric pressure was recorded.

ANALYSIS

For the long distending periods at MDP $+2 \mathrm{~mm} \mathrm{Hg}$ before and after drug administration, gastric tone was measured as the mean balloon volume over consecutive five minute intervals. For each two minute distending period, the dependent variable was measured by averaging the recordings. The perception score corresponding to each distension was computed. Perception threshold was defined as the first level of pressure (during isobaric distensions) or volume (during isovolumetric distensions) that evoked a perception score of 1 or more. Discomfort threshold was defined as the first level of pressure (during isobaric distensions) or volume (during isovolumetric distensions) that provoked a perception score of 5 or more. The gastric wall tension at the threshold for first perception and for discomfort was calculated using Laplace's law. ${ }^{12}$ Pressure-volume and pressure-perception curves were obtained from the stepwise distensions. Different curve models (including linear, parabolic, sigmoid, hyperbolic, and power exponential models) were evaluated for goodness of fit of the individual pressurevolume and pressure-perception curves. A linear regression model provided the best fit (median $r^{2}=94.1 \%$ ).

To evaluate the effect of sumatriptan on basal intragastric volume, the average volumes calculated over a 45 minute period before and after administration of the drug were compared using the paired Student's $t$ test. To evaluate the effect of sumatriptan on phasic contractility, which corresponds to slow changes in baseline volume after filtering out respiratory artefact, a baseline reconstruction was performed using a computerised algorithm. The baseline was calculated on the basis of an estimated weighted moving average (EWMA): $\mathrm{b}_{\mathrm{r}+1}=\delta \times$ $\mathrm{c}_{\mathrm{r}+1}+(1-\delta) \times \mathrm{b}_{\mathrm{r}}$

where: $b_{r}$ and $b_{r+1}$ are the actual and the next baseline coefficient; $\mathrm{c}_{\mathrm{r}+1}$ is the signal value at time constant $\mathrm{r}+1$; and $\delta$ is the smoothing constant.

The constant $\delta$ is a value between 0 and 1 . With $\delta=0$, the baseline will be a constant; with $\delta=1$, the baseline and the recorded signal will be identical. By selecting the correct value for $\delta$, according to the signal, a temporary baseline is calculated. The disadvantage of this method is that the baseline depends on earlier data samples only. To overcome this, a second temporary baseline was calculated, depending only on later data samples: $\mathrm{b}_{\mathrm{r}-1}=\delta \times \mathrm{c}_{\mathrm{r}-1}+(1-\delta) \times$ $b_{r}$. The final baseline is the mean of the two temporary baselines.

Consecutively, a motility index (MI) was calculated as the area between the signal and the baseline normalised over time. This normalisation allowed comparison of periods of different length. MI values before and after sumatriptan were compared using the paired Student's $t$ test. Previously, we observed that phasic fundus contractions, reflected in the MI, are not related to antral contractile activity (unpublished observations). 


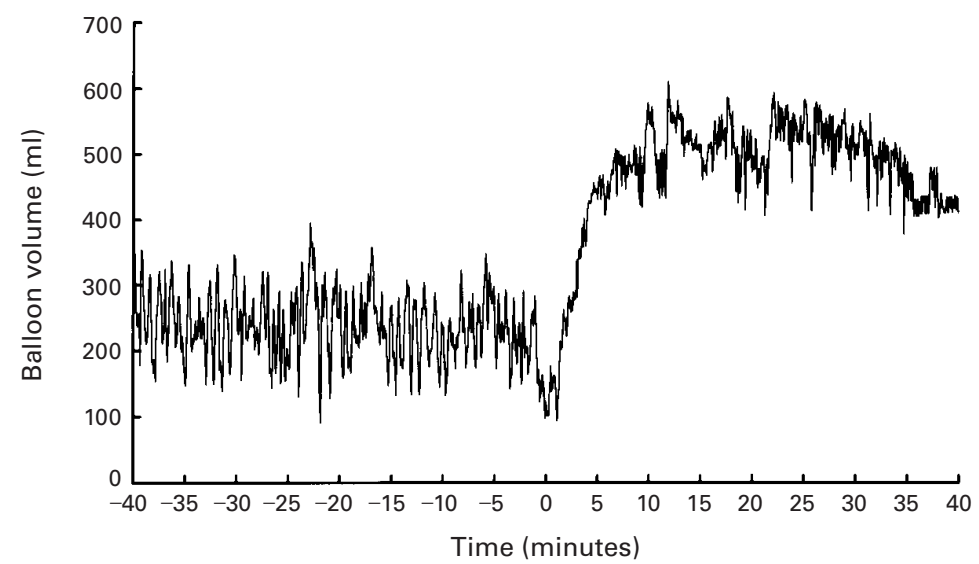

Figure 1 Representative tracing of intragastric volume, measured by a barostat, before and after administration of sumatriptan $6 \mathrm{mg}$ subcutaneously at time 0 .

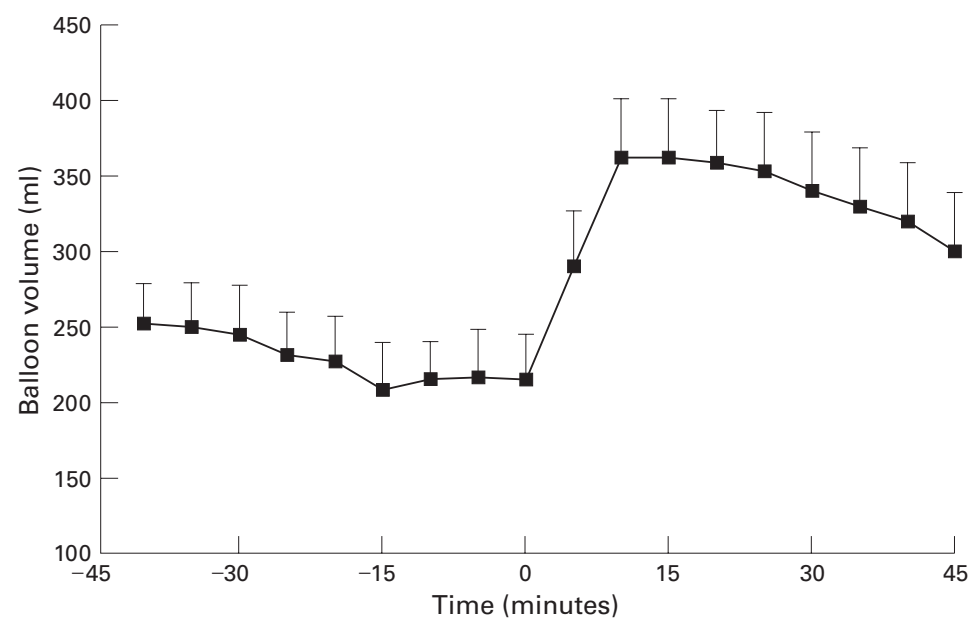

Figure 2 Mean intragastric volume $(n=11)$ at five minute intervals as measured with the barostat, before and after administration of sumatriptan $6 \mathrm{mg}$ subcutaneously at time 0.

To evaluate the effect of sumatriptan on gastric compliance and perception of gastric distension, the intercepts and slopes, obtained by linear regression analysis of pressurevolume curves and distension-perception curves, were compared by Student's $t$ test.

To evaluate the effect of sumatriptan on perception and discomfort thresholds, the number of subjects that reported perception or discomfort at a given intragastric volume or pressure, were compared before and after drug administration using a logistic regression procedure with stratification, implying exact conditional inference. In addition, the perception and discomfort thresholds before and after sumatriptan were compared using the paired Student's $t$ test. The relation between changes in perception and changes in gastric tone was analysed by linear regression analysis of the gastric relaxation and the increase in perception thresholds after drug administration in the second protocol. Differences were considered to be significant at the $5 \%$ level.

\section{Results}

INFLUENCE OF PLACEBO ON GASTRIC FUNDUS TONE AND ON THE PERCEPTION OF GASTRIC DISTENSION

The effect of placebo on fundic tone was assessed in ten volunteers, in whom the MDP was $7.7(0.5) \mathrm{mm} \mathrm{Hg}$. The average intragastric volume at $\mathrm{MDP}+2 \mathrm{~mm} \mathrm{Hg}$, as measured by the barostat during the basal 45 minute period was 236 (34) ml. After the administration of placebo, the average intragastric volume remained unchanged (220 (32) $\mathrm{ml}, \mathrm{NS}$ ). Phasic contractile activity of the proximal stomach, assessed by calculating MI, was also not altered by administration of placebo (MI 36.1 (4.3) versus 39.9 (3.4) $\mathrm{ml} \times$ sec; $\mathrm{NS}$ ).

Administration of placebo in ten healthy subjects had no significant influence on the pressure level inducing threshold perception (10.7 (0.6) versus $11.5(0.7) \mathrm{mm} \mathrm{Hg}, \mathrm{NS}$ ) or discomfort (19.1 (1.1) versus 18.1 (1.1) $\mathrm{mm}$ $\mathrm{Hg}$, NS) during isobaric distensions. The slope (50 (4) versus $50(4.8) \mathrm{ml} / \mathrm{mm} \mathrm{Hg}$, NS) and the y intercept (146 (50) versus 119 (35) $\mathrm{ml}$, NS) of the pressure-volume curve, obtained after linear model fitting, were also not altered. Likewise, administration of placebo in seven healthy subjects had no significant influence on the volumes inducing threshold perception (291 (53) versus 277 (33) ml, NS) or discomfort (700 (63) versus 682 (38) $\mathrm{ml}, \mathrm{NS}$ ) during isovolumetric distensions.

EFFECT OF SUMATRIPTAN ON GASTRIC FUNDUS TONE

The effect of sumatriptan on fundic tone was assessed in 11 volunteers, in whom the MDP was $6.5(0.5) \mathrm{mm} \mathrm{Hg}$. The average intragastric volume at $\mathrm{MDP}+2 \mathrm{~mm} \mathrm{Hg}$, as measured by the barostat during the basal 45 minute period was 229 (25) $\mathrm{ml}$. In all volunteers, subcutaneous administration of sumatriptan caused an immediate relaxation of the gastric fundus, reflected by an increase in the balloon volume within five minutes after administration (fig 1). The maximum increase in balloon volume was 209 (39) $\mathrm{ml}(\mathrm{p}=0.0004)$, and this occurred 10-15 minutes after the administration of sumatriptan. The intragastric volume remained significantly increased until the end of the measuring period (fig 2). The mean balloon volume averaged over 45 minutes increased from 229 (25) $\mathrm{ml}$ to 335 (34) $\mathrm{ml}$ after sumatriptan $(p=0.002)$.

The effect of sumatriptan on phasic contractile activity of the fundus was assessed by calculating MI. In all volunteers, sumatriptan caused a significant inhibition of phasic contractions, expressed as a significant decrease of the MI (44.4 (4.9) versus 24.8 (3) $\mathrm{ml} \times \mathrm{sec}$; $\mathrm{p}<0.001)$.

\section{EFFECT OF SUMATRIPTAN ON ISOBARIC GASTRIC} DISTENSIONS

Both before and after the administration of sumatriptan, distensions of the stomach with progressively higher set pressures produced progressively larger intragastric volumes. At the same distending pressures, intragastric volumes after sumatriptan were significantly larger than the corresponding volumes prior to drug administration (fig 3). The slope of the pressure-volume curves, obtained after linear model fitting, was not altered by sumatriptan (51.4 (4.7) versus 54.3 (6.4) $\mathrm{ml} / \mathrm{mm} \mathrm{Hg}$, NS). The y intercept of the pressure-volume curves 


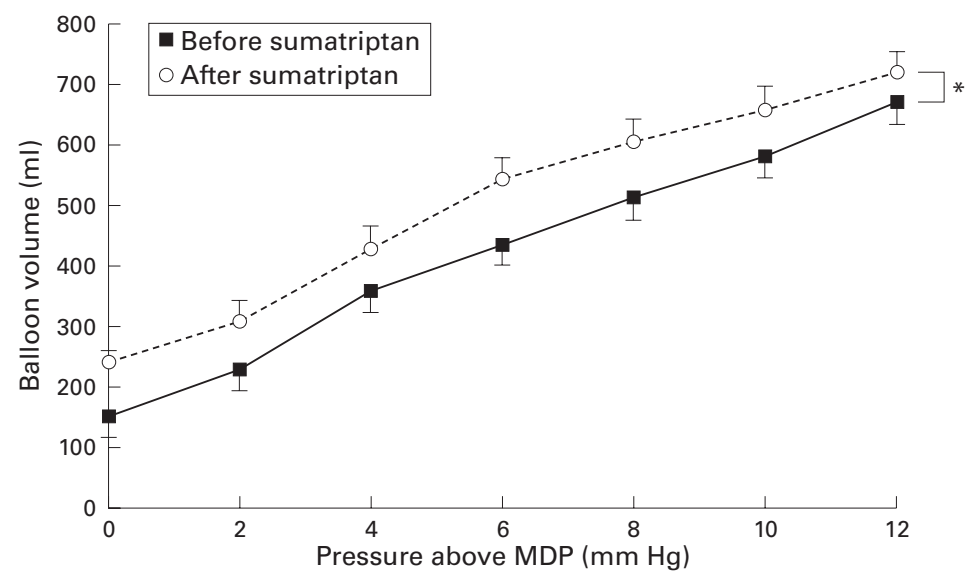

Figure 3 Pressure-volume relation obtained by gradually increasing isobaric gastric distensions before and after sumatriptan $6 \mathrm{mg}$ subcutaneously $(n=13)$. Linear model fitting revealed that sumatriptan causes a shift of the pressure-volume curve to significantly higher volumes $(p<0.05)$. MDP, minimal distending pressure.

was significantly increased after sumatriptan (191 (37) versus 275 (38) $\mathrm{ml}, \mathrm{p}=0.01$ ). This shift of the pressure-volume curve towards higher volumes probably reflects the sumatriptan induced relaxation of the gastric fundus.

Sumatriptan did not change the average perception score at the same distending pressures (fig 4A). After linear model fitting, both the slope and the $\mathrm{x}$ intercept of the individual pressure-perception curves were unaltered (respectively $0.33(0.06)$ versus $0.32(0.07)$ per $\mathrm{mm} \mathrm{Hg}$, and $0.8(0.4)$ versus $1.0(0.9) \mathrm{mm} \mathrm{Hg}$, NS). Also, sumatriptan did not change the pressure level inducing threshold perception (11 (0.8) versus 12.7 (1.3) $\mathrm{mm} \mathrm{Hg}$, NS) or discomfort (17 (1) versus 17.9 (1.2) $\mathrm{mm} \mathrm{Hg}$, NS). This was confirmed by logistic regression analysis of pressure-perception curves and pressure-discomfort curves before and after sumatriptan (both NS). However, the corresponding volumes at the threshold for perception (403 (52) versus 513 (61) $\mathrm{ml}, \mathrm{p}<0.001)$ and at the threshold for discomfort (662 (62) versus 733 (54) $\mathrm{ml}, \mathrm{p}<0.01$ ) were significantly increased by sumatriptan. The relation between the increase of the intraballoon volume at 15 minutes and the increase in volumes inducing first perception was significant $(r=0.53, \mathrm{p}<0.05)$. There was a highly signifi- cant relation between the increase of the intraballoon volume at 15 minutes and the increase in volumes inducing discomfort $(r=0.81$, $\mathrm{p}<0.005)$.

EFFECT OF SUMATRIPTAN ON ISOVOLUMETRIC

GASTRIC DISTENSIONS

Both before and after the administration of sumatriptan, distensions of the stomach with progressively larger volumes produced progressively larger intragastric pressures. After linear model fitting, both the slope and the y intercept of the individual volume-pressure curves were not significantly altered (respectively $1.4(0.1)$ versus $1.2(0.2) \mathrm{mm} \mathrm{Hg} / 100 \mathrm{ml}$ and 5.7 (0.6) versus $5.3(0.6) \mathrm{mm} \mathrm{Hg}$, NS).

Sumatriptan significantly decreased the average perception score at the same distending volumes (fig $4 \mathrm{~B}$ ). The slope of the volumeperception curves, obtained after linear model fitting, was not altered by sumatriptan (0.7 $(0.1)$ versus $0.6(0.1)$ per $100 \mathrm{ml}, \mathrm{NS})$. The $\mathrm{x}$ intercept of the volume-perception curves was significantly higher after sumatriptan (124 (32) versus $286(35) \mathrm{ml}, \mathrm{p}<0.001)$. In addition, sumatriptan caused a significant increase of the volume inducing first perception (300 (31) versus 443 (127) $\mathrm{ml}, \mathrm{p}=0.008$ ). The increase in the volume inducing discomfort did not reach significance (814 (51) versus 929 (78) $\mathrm{ml}, \mathrm{NS}$ ). Logistic regression analysis of both volumeperception curves and volume-discomfort curves showed a significant shift towards higher volumes after sumatriptan $(p<0.001$ and $\mathrm{p}<0.03$ respectively) (fig 5 ). The corresponding pressures at the threshold for perception (9.5 (0.9) versus $10.2(1.2) \mathrm{mm} \mathrm{Hg}, \mathrm{NS}$ ) and at the threshold for discomfort (17.8 (1.8) versus 16.7 (1.7) $\mathrm{mm} \mathrm{Hg}, \mathrm{NS}$ ) were not significantly altered by sumatriptan.

\section{EFFECT OF SUMATRIPTAN ON PERCEPTION AND} DISCOMFORT THRESHOLDS

Pooling of the responses to isobaric and isovolumetric distensions (20 subjects; 13 men and seven women) confirmed that the threshold for perception was reached at higher volumes (367 (37) versus 489 (43) $\mathrm{ml}$, $\mathrm{p}=0.00001)$, but at the same pressures $(10.5$ (0.6) versus 11.8 (1) $\mathrm{mm} \mathrm{Hg}$, NS) after sumatriptan. Likewise, sumatriptan caused a significant increase of the volume (716 (46)
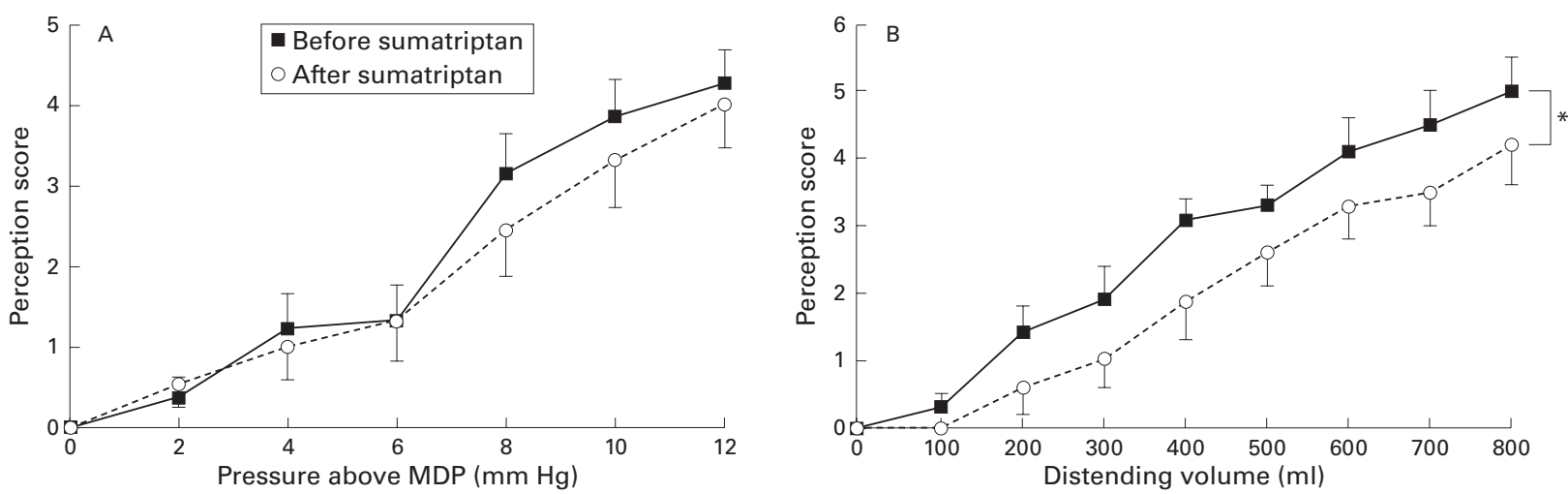

Figure 4 (A) Corresponding mean perception scores for gradually increasing isobaric distentions before and after sumatriptan 6 mg subcutaneously $(n=13)$. (B) Corresponding mean perception scores for gradually increasing isovolumetric distensions before and after sumatriptan 6 mg subcutaneously $(n=7)$. MDP, minimal distending pressure. 


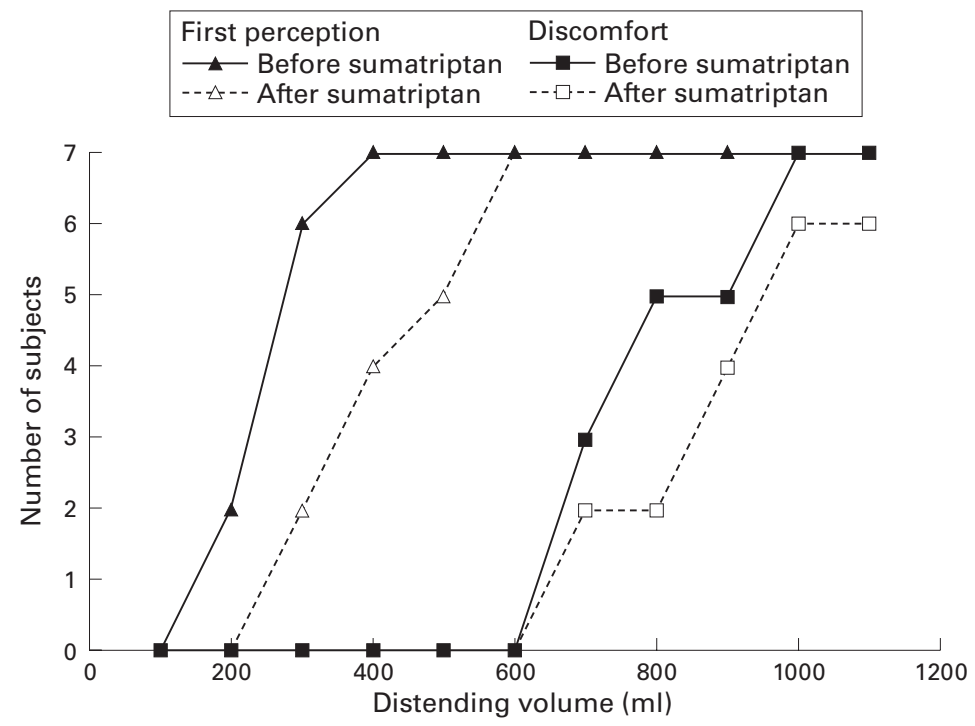

Figure 5 Number of subjects that reported first perception at a given distending volume before and after sumatriptan $6 \mathrm{mg}$ subcutaneously, and number of subjects that reported discomfort at a given distending volume before and after sumatriptan 6 mg subcutaneously $(n=7)$. Sumatriptan induced a significant shift of the volume-perception curve and of the volume-discomfort curve towards higher volumes ( $p<0.005$; logistic regression).

versus 801 (48) $\mathrm{ml}, \mathrm{p}=0.005)$, but not of the pressure (17.3 (0.9) versus $17.5(0.9) \mathrm{mm} \mathrm{Hg}$, NS) at which the threshold for discomfort was reached. After sumatriptan, the gastric wall tension at the threshold for perception and at the threshold for discomfort were significantly increased (25.7 (2.3) versus 32.4 (2.6), and $57.2(4.8)$ versus $61.4(4.9) \mathrm{cm} \times \mathrm{mm} \mathrm{Hg}$ respectively, $\mathrm{p}<0.05)$.

\section{Discussion}

In the present study, we have shown that administration of the $5-\mathrm{HT}_{1}$ receptor agonist sumatriptan in the interdigestive state in man causes an immediate and profound relaxation of the gastric fundus. Phasic contractions of the gastric fundus are also inhibited by the administration of sumatriptan. After the administration of sumatriptan, larger intragastric volumes are present at the same intragastric pressure. Moreover, sumatriptan allows larger volumes to be accommodated before thresholds for perception or discomfort are reached. After sumatriptan, calculated wall tensions at the thresholds for perception and discomfort are significantly lower.

The mechanism by which sumatriptan influences fundus tone in man is not known, but several possible pathways can be involved. The fact that sumatriptan poorly penetrates the blood-brain barrier ${ }^{10}$ argues against an action at the level of the central nervous system. In man, administration of glucagon or somatostatin analogue also causes a relaxation of the gastric fundus. ${ }^{12}$ Previous measurements of plasma somatostatin and glucagon concentrations before and after the administration of sumatriptan, rule out release of somatostatin or glucagon as a mechanism by which sumatriptan might induce a fundic relaxation. ${ }^{15}$

In man, the effect of 5-HT on gastric fundus tone has not been studied directly. Our observation that sumatriptan causes a relaxation of the gastric fundus in man is consistent with animal studies that observed 5-HT induced relaxations of the stomach, through the activation of intrinsic inhibitory neurones. ${ }^{45}$ Several subtypes of 5-HT receptors are known. ${ }^{6}$ Of these, $5-\mathrm{HT}_{1 \mathrm{~A}}, 5-\mathrm{HT}_{1 \mathrm{P}}$, and $5-\mathrm{HT}_{3}$ receptors have been identified in the enteric nervous system of the stomach. ${ }^{7}$ Selective antagonism of $5-\mathrm{HT}_{3}$ receptors did not influence interdigestive and postprandial fundus tone in $\operatorname{man}^{89}$ and sumatriptan has little or no affinity at $5-\mathrm{HT}_{3}$ receptors. ${ }^{6}$ In the myenteric plexus of the stomach, presynaptic $5-\mathrm{HT}_{1 \mathrm{~A}}$ receptors mediate the inhibition of transmitter release from cholinergic nerve endings. ${ }^{7}$ Activation of a presynaptic $5-\mathrm{HT}_{1 \mathrm{~A}}$ receptor on cholinergic motor neurones would result in decreased acetylcholine release. However, sumatriptan is only a weak agonist at $5-\mathrm{HT}_{1 \mathrm{~A}}$ receptors. ${ }^{6}$ Moreover, in man, it was reported that atropine $(6 \mu \mathrm{g} / \mathrm{kg} / \mathrm{h}$ ) did not change fasting gastric tone. ${ }^{16}$ These observations seem to argue against activation of enteric neuronal $5-\mathrm{HT}_{1 \mathrm{~A}}$ receptors underlying the effects of sumatriptan on gastric fundus tone in man.

In the guinea pig, the 5-HT induced gastric relaxation seems to be mediated via the release of nitric oxide through activation of a $5-\mathrm{HT}_{1}$ like receptor. ${ }^{5}$ In in vitro studies in the guinea pig, we showed that sumatriptan is an agonist at $5-\mathrm{HT}_{1 \mathrm{P}}$ receptors on enteric neurones. ${ }^{11}$ $5-\mathrm{HT}_{1 \mathrm{P}}$ receptors are mainly present on nitrergic neurones in the myenteric plexus of the guinea pig stomach, where they mediate a prolonged depolarisation in response to the application of $5-\mathrm{HT}^{7}{ }^{17}$ Animal studies that observed 5-HT induced relaxations of the stomach, through the activation of intrinsic inhibitory neurones, are in agreement with the hypothesis that the $5-\mathrm{HT}_{1 \mathrm{P}}$ receptor is located on inhibitory motor neurones. ${ }^{45}$ Antagonists at this receptor include the prokinetic benzamides such as renzapride or cisapride..$^{7819}$ It has indeed been shown that cisapride, an antagonist at the $5-\mathrm{HT}_{1 \mathrm{P}}$ receptor, is able to inhibit 5-HT induced relaxation of the guinea pig stomach. ${ }^{5}$ Using gastric barostat studies in cats in vivo, we were able to confirm that the sumatriptan induced relaxation of the gastric fundus is mediated via the release of nitric oxide. ${ }^{20}$ Thus, we hypothesise that the effects of sumatriptan on gastric fundus tone in man might reflect the activation of enteric neuronal $5-\mathrm{HT}_{1 \mathrm{P}}$ receptors on intrinsic nitrergic neurones.

In the present study, we also showed that sumatriptan causes a significant increase in the volumes needed to reach the thresholds for perception or discomfort. In contrast, the pressures at the thresholds for perception or discomfort are not altered by sumatriptan. Visceral sensations are modulated at different levels of the brain/gut axis ${ }^{21}$ and, theoretically, sumatriptan could be acting at each of these levels. It seems less likely that sumatriptan alters perception at a central level, since it penetrates poorly the blood-brain barrier. ${ }^{22} \mathrm{Su}-$ matriptan may alter the perception of gastric distension because of its effect on gastric tone, or because it has an antinociceptive effect on the afferent sensorial pathway. Recently, 
Notivol et al showed that gastric tone is a major determinant of the sensitivity to gastric distension. ${ }^{12}$ We observed that the sumatriptan induced decrease in gastric tone is accompanied by higher intragastric volumes and higher calculated wall tensions at the thresholds for perception and discomfort during gastric distension. In a recent study, the use of a tensostat showed that perception of gastric distension before and during glucagon induced gastric relaxation was determined by gastric wall tension. ${ }^{23}$ The present study, which used a barostat, does not allow a clear separation of the effects on tone from the effects on perception. However, the significant relation between the sumatriptan induced gastric relaxation and the increase in intragastric volumes needed to induce perception or discomfort suggests that the decrease in tone is the principal effect of sumatriptan, and that higher volume thresholds are most likely occurring secondary to the sumatriptan induced relaxation.

The current study may have important implications for the treatment of patients with functional dyspepsia. Recent studies showed that the accommodation of the gastric fundus to a meal, measured by a barostat, is impaired in a subgroup of patients with functional dyspepsia, ${ }^{23-25}$ and that this is associated with a high prevalence of early satiety and weight loss. ${ }^{25}$ In these patients, administration of sumatriptan is able to restore gastric accommodation to a meal and to improve symptoms of early satiety, confirming the therapeutic potential of $5-\mathrm{HT}_{1 \mathrm{P}}$ receptor agonists in functional dyspepsia. ${ }^{26}$ In addition, enhanced perception of both isobaric and isovolumetric gastric distension has been reported in functional dyspepsia, although the compliance of the gastric wall is not different. ${ }^{27}{ }^{28}$ It has been suggested that altered perception of the meal induced gastric distension may be a major mechanism of symptom production in functional dyspepsia. Sumatriptan causes a significant increase in the volumes needed to reach the thresholds for discomfort, but does not alter the pressure needed to reach discomfort. Further studies will be required to investigate whether sumatriptan, or other $5-\mathrm{HT}_{1}$ receptor agonists, may also have a potential therapeutic effect in dyspeptic patients with hypersensitivity to gastric distension.

In conclusion, the present study showed that administration of the 5-HT 1 receptor agonist sumatriptan in man induces an immediate and profound relaxation of the gastric fundus. After the administration of sumatriptan, larger intragastric volumes are present at the same intragastric pressure. Moreover, sumatriptan allows larger volumes to be accommodated before thresholds for perception or discomfort are reached. The pressures needed to reach thresholds for perception or discomfort during gastric distensions are not altered by sumatriptan. Based on in vitro studies, we hypothesise that sumatriptan is acting at enteric neuronal $5-\mathrm{HT}_{1 \mathrm{P}}$ receptors to cause these effects. Confirmation of this hypothesis awaits the availability of suitable ligands. The effects of sumatriptan on the gastric fundus may have therapeutic potential in the treatment of patients with functional dyspepsia.

1 Azpiroz F, Malagelada JR. Importance of vagal input in maintaining gastric tone in the dog. $f$ Physiol 1987;384:511-24.

2 Azpiroz F, Malagelada JR. Vagally mediated gastric relaxation induced by intestinal nutrients in the dog. $A m \mathcal{F}$ Physiol 1986;251:G727-35.

3 Desai KMW, Sessa WC, Vane JR. Involvement of nitric oxide in the reflex relaxation of the stomach to accommodate food or fluid. Nature 1991;351:477-9.

4 Bulbring E, Gershon MD. 5-Hydroxytryptamine participation in the vagal inhibitory innervation of the stomach. 7 Physiol 1967;192:823-46.

5 Meulemans AL, Helsen LF, Schuurkes JAJ. The role of nitric oxide (NO) in 5-HT induced relaxations of the guinea-pig stomach. Naunyn Schmiedebergs Arch Pharmacol 1993;384:424-30.

6 Hoyer D, Clarke DE, Fozard JR, et al. International Union of Pharmacology classification of receptors for 157-203.

7 Tack JF, Janssens J, Vantrappen G, et al. Actions of 5-hydroxytryptamine on myenteric neurones in the gastric antrum of the guinea-pig. Am f Physiol 1992;263:G838-46.

8 Zerbib F, Bruley des Varannes S, Oriola RC, et al. Alosetron does not affect the visceral perception of gastric distension in healthy subjects. $A$

9 Reference withdrawn. pharmacodynamic and pharmacokinetic properties, and therapeutic efficacy in the acute treatment of migraine and cluster headache. Drugs 1992;43:776-98.

11 Reference withdrawn

12 Notivol R, Coffin B, Azpiroz F, et al. Gastric tone determines the sensitivity of the stomach to distension. Gastroenterology 1995;108:330-6.

13 Gregersen H, Kassab, G. Biomechanics of the gastrointestinal tract. Neurogastroenterol Motil 1996;8:277-97.

14 Bourgeois S, Coulie B, Tack J, et al. The somatostatinanalogue octreotide inhibits tone and phasic contractile activity of the gastric fundus in man [abstract]. Gastroenteractivity of the gastric func

15 Coulie B, Tack J, Bouillon R, et al. 5-Hydroxytryptamine-1 receptor activation inhibits endocrine pancreatic secretion in man. Am f Physiol 1998;37:E317-20.

16 Bruley Des Varannes S, Parys V, Ropert A, et al. Erythromycin enhances fasting and postprandial proximal gastric tone in humans. Gastroenterology 1995;109:32-9.

17 Michel K, Sann H, Schaaf C, et al. Subpopulations of gastric myenteric neurons are differentially activated via distinct serotonin receptors: projection, neurochemical coding, and functional implications. $\mathcal{F}$ Neurosci 1997;17:8009-17.

18 Nemeth PR, Ort CA, Zafirov DH, et al. Interactions between serotonin and cisapride on myenteric neurones. Eur F Pharmacol 1985;108:77-83.

19 Mawe GM, Branchek TA, Gershon MD. Blockade of 5-HT mediated enteric slow EPSPs by BRL 24924: gastrokinetic mediated enteric slow EPSPs by BRL 24924
effects. Am $\mathcal{F}$ Physiol 1989;257:G386-96.

20 Coulie B, Tack J, Sifrim D, et al. Role of nitric oxide in fasting gastric fundus tone and in 5-hydroxytryptamine-1 receptor-mediated relaxation of the gastric fundus. Am f Physiol 1999;276:G373-7.

21 Mayer EA, Gebhart GF. Basic and clinical aspects of visceral hyperalgesia. Gastroenterology 1994;107:271-93.

22 Sleight AJ, Cervenka A, Peroutka SJ. In vivo effects of sumatriptan (GR 46175) on extracellular levels of 5-HT in the guinea-pig. Neuropharmacology 1991;29:511-13.

23 Distrutti E, Azpiroz F, Soldevilla A, et al. Gastric wall tension determines perception of gastric distention. Gastroenterology 1999;116:1035-42.

24 Coffin B, Azpiroz F, Guarner F, et al. Selective gastric hypersensitivity and reflex hyporeactivity in functional dyshypersensitivity and reflex hyporeactivity in
pepsia. Gastroenterology 1994;107:1345-51.

25 Troncon LEA, Thompson DG, Ahluwalia NK, et al. Relations between upper abdominal symptoms and gastric distension abnormalities in dysmotility like functional dyspepsia and after vagotomy. Gut 1995;37:17-22.

26 Tack J, Piessevaux H, Coulie B, et al. Role of impaired gastric accommodation to a meal in functional dyspepsia. Gastroenterology 1998;115:1346-52.

27 Mearin F, Cucala M, Azpiroz F, et al. The origin of symptoms on the brain-gut axis in functional dyspepsia. Gastroenterology 1991;101:999-1006.

28 Bradette M, Pare P, Douville P, et al. Visceral perception in health and functional dyspepsia. Crossover study of gastric distensions with placebo and domperidone. Dig Dis Sci 1991;36:52-8. 